\title{
Coates' Algorithm
}

\section{Introduction}

In this chapter, we consider the problem of finding a function with a certain set of poles. That this problem is non-trivial in the case of algebraic functions (although it is trivial in the case of rational functions) can be seen from the fact that such functions need not always exist. For example, on the curve defined by $\sqrt{X^{3}+1}$, there is no function with a zero of order 1 at one place lying over the point $X=0$ and a pole of order 1 at infinity and no other poles or zeros, but there is one with divisor 3 times that (i.e. the divisor has order 3). On the curve defined by $Y^{2}=X^{3}-3 X^{2}+X+1$, there are no functions with a zero at one place lying over $X=0$ and a pole at the other, both having the same order, and no other zeros or poles (see Appendix 2 example 7).

Coates published ${ }^{*}$ an algorithm in 1970 (Coates,1970) to find functions on algebraic curves over algebraic number fields as part of a bigger project on Diophantine equations (Baker \& Coates, 1970), and this context coloured his precise formulation of the results he stated and proved. We will wish to use a slightly different statement of the algorithm in a slightly different context, as described below. In particular, in view of the remarks in the last chapter, we can assume that no place at which we wish to compute is ramified, although the theory only requires this to be true of infinity (and, even if this is not so, Coates (1970) Theorem 2 implies that a slightly modified result is still true). For computational convenience, we will often use an unramified representation even when the theory does not require it.

* Coates only stated his algorithm for algebraic number fields, and was only interested in showing that it was mathematically effective. The extension to arbitrary ground fields is, I believe, original (though not profound). I also believe that this work describes the first computer implementation of Coates' Algorithm. 
The Algorithm

Let $D$ be a divisor of poles (i.e. all multiplicities in it are negative) all of whose places are finite. Then the algorithm will produce a basis for the $K$ vector space of all functions $f$ with $(f) \geq D$, i.e. the space of functions with poles no worse than $D$. This algorithm relies on two subsidiary ones, INTEGRAL_BASIS_REDUCTION and NORMAL_BASIS_REDUCTION, which, following the dictates of the top-down programmers, are described after the main algorithm. These algorithms are described in simplified form, in that we do not mention the complications caused by the necessity to take representations of our curve on which certain places are unramified. We also do not describe several of the techniques used for efficiency, since they complicate the algorithms unnecessarily for our purposes, even though they are important from a practical point of view.

The algorithm can be summarised as follows: we start with any list of functions which has no finite poles, progressively insert all the finite poles as specified by $D$ (using INTEGRAL_BASIS_REDUCTION), and then find those resulting functions that have no poles over infinity (using NORMAL BASIS_REDUCTION).

Before we can define this algorithm fully, we need a couple of definitions. Let $V$ be a module over the (commutative) ring $R$. We say that $\left(v_{1}, \ldots, v_{k}\right)$ form a basis for $V$ over $R$ if all the $v_{i}$ lie in $V$ and each $v$ in $V$ can be expressed in the form $r v=\sum_{i=1}^{k} r_{i} v_{i}$ where $r$ and the $r_{i}$ are in $R$, with $r$ non-zero, and all such expressions are unique up to multiplication by elements of $R$. A basis is said to be an integral basis iff $r$ can be chosen to be 1 in all such expressions, i.e. each element of $V$ can be expressed as a linear combination of the $v_{i}$ with coefficients in $R$. 


\section{COATES}

Input:

$F(X, Y)$ : the equation of the curve

(possibly in a multivariate representation, e.g. $F(X, Y)=0, G(X, Y, Z)=0$ ).

D : a divisor of finite poles on the curve

\section{Output:}

V : A basis for the $K$-space of functions $f:(f) \geq D$

[1] $\mathrm{V}:=\left(1, \mathrm{Y}, \mathrm{Y}^{* *} 2 \ldots \mathrm{Y}^{* *}(\right.$ degree of $\mathrm{F}$ in $\left.\mathrm{Y}-1)\right)$

In the event of a multivariate representation, we let $V$ be the set of all possible products of the algebraic variables raised to powers less than their degree.

$V$ is now a basis (but not an integral basis - see above for a precise definition of this term) for the $K[X]$ module of functions with no finite poles worse than $D$ (or indeed worse than 0 ).

[2] For Each $P$ over which places in $D$ lie $\mathrm{V}:=$ INTEGRAL_BASIS_REDUCTION(V, P, that part of D which lies over P).

Each step of this makes $V$ have the right multiplicities at those places of $D$ which lie over $P . \quad V$ is now a basis for the $K[X]$ module of functions with no finite poles worse than those in $D$ and which is "quasi-integral" in the following sense: any member of that module can be expressed as a linear combination of elements in $V$ with coefficients from $K[X]$, possibly divided by a member of $K[X]$, but this member of $K[X]$ will have no zeros at the (finite) places which occur in $D$.

[3] Now ensure that this really is an integral basis.

[3.1] Z:=An arbitrary element of K (e.g. a "GENSYM").

[3.2] $\mathrm{A}:=$ determinant $\mathrm{A}(\mathrm{I}, \mathrm{J})$, where

$$
A(I, J)=\text { coefficient } X^{* *} 0 \text { in } V(I) \text { expanded about } J^{\prime} \text { th place over } Z .
$$


[3.3] For all roots (in $\mathrm{Z}$ ) of the numerator or denominator of $\mathrm{A}$ $\mathrm{V}:=$ INTEGRAL_BASIS_REDUCTION(V, Z, 0 divisor over $\mathrm{Z})$.

[4] V:=NORMAL_BASIS_REDUCTION (V,places of curve lying over infinity).

This ensures that, not only do we have an integral basis, but that its elements are of as high an order (i.e. have as little of a pole) as possible at infinity. The elements of this basis $V$, when multiplied by suitable powers of $X$, are the required basis

[5] $V:=$ For Each $V(I)$ with order $N \geq 0$ at infinity

Collect $(\mathrm{V}(\mathrm{I}), \mathrm{V}(\mathrm{I}) * \mathrm{X}, \ldots \ldots, \mathrm{V}(\mathrm{I}) * \mathrm{X} * * \mathrm{~N})$.

In fact $N$ must be the minimum order of $\mathrm{V}(\mathrm{I})$, over all places lying over infinity.

I have currently no computing time analysis for this algorithm, but in cases arising in practice, step 3 is nearly always the most expensive operation. This step is needed to ensure that we have not inadvertently started with, or introduced later on, a dependence between the elements of $V$ over some point $Z$ which did not appear in the divisor $D$. Under many special formulations of the problem one can prove that this cannot happen, and I conjecture that there is some wide class of problems for which this is true, and that it should be possible to reduce all problems to one of this kind. However, I have not made much progress in this area.

If one excepts step 3 , then it might appear that the time would be proportional to the sum of the multiplicities in the divisor, since this is the number of individual INTEGRAL_BASIS_REDUCTION steps that are performed, to within some complicated edge effects. Things are not quite so simple, however, because such steps tend to increase the size of the elements of $V$. I believe that the time is, in fact, exponential in the sum of the multiplicities, but the underlying analysis is not rigorous. The dependence of the time on the degree of $F(X, Y)$ is clearly much worse, but here again $I$ have no definite formula. One of the unfortunate features of this algorithm is that one starts with a fairly simple basis in step 1, step 2 produces an integral basis with enormous expressions, and step 4 then reduces them drastically in size, often to the same complexity as the original basis. Thus it would appear that there is a much better formulation of this fundamental 
algorithm waiting to be discovered. Sometimes performing reduction steps at infinity (which can be done in the middle of reduction steps at other points) can control this growth, but frequently it merely wastes time (since the reduction at infinity must be performed at the end as well) and does not reduce the expression size at all.

\section{INTEGRAL_BASIS_REDUCTION}

Input:

$V$ : a basis for the $K[X]$ module.

P: a value of $X$ to ensure that the basis is integral at.

D: a divisor of poles lying wholly over $P$.

\section{Output}

V: a modified basis, integral over $P$.

[1] Let $A(I, J)$ be the coefficient of $(X-P)^{* *} N(J)$ in the expansion of $V(I)$ about the $J$ 'th place in $D$, where $N(J)$ is the multiplicity with which that place occurs.

There might be problems if $(X-P)$ is not a local parameter, so in fact we may need to consider some fractional power of $(X-P)$. However, the algorithm is easier to describe without this complication.

[2] While A is singular do:

[2.1] Let $\mathrm{B}(\mathrm{I})$ be elements of $K$ such that $\mathrm{SUM}(\mathrm{B}(\mathrm{I}) * \mathrm{~A}(\mathrm{I}, \mathrm{J}))=0$ for all $\mathrm{J}$, but the $\mathrm{B}(\mathrm{I})$ are not all zero. In particular, assume $B(K)$ is non-zero.

[2.2] $\mathrm{V}(\mathrm{K}):=\operatorname{SUM}\left(\mathrm{B}(\mathrm{I})^{*} \mathrm{~V}(\mathrm{I})\right) /(\mathrm{X}-\mathrm{P})$

Then the $\mathrm{V}(\mathrm{I})$ are still linearly independent over $K(X)$ and have poles no worse than $D$ at $P$.

[2.3] Recompute $A(K, J)$ as in [1].

[3] Return V 


\section{NORMAL_BASIS REDUCTION}

Input:

$\mathrm{V}$ : an integral basis for the $K[X]$ module

D: The places of the curve lying over infinity.

\section{Output}

$\mathrm{V}$ : A normal integral basis for the $\mathrm{K}[\mathrm{X}]$ module

[1] Sort the $V(I)$ in decreasing order of their minimum order at all of the places over infinity.

[2] Let $A(I, J)$ be the coefficient of $1 / X^{* *} 0$ in the expansion of $V(I)$ about the $J$ 'th place in D.

There might be problems if $1 / \mathrm{X}$ is not a local parameter, so in fact we may need to consider some fractional power of $1 / \mathrm{X}$. However, the algorithm is easier to describe without this complication.

[3] While $\mathrm{A}$ is singular do:

[3.1] Let $B(I)$ be elements of $K$ such that $\operatorname{SUM}\left(B(I)^{*} A(I, J)\right)=0$ for all $J$, but the $B(I)$ are not all zero. In particular, assume $\mathrm{B}(\mathrm{K})$ is non-zero.

Among all such possible $B(I)$, we want the one involving the $V(I)$ of highest order, i.e. the first one to be discovered if one tries Gaussian elimination without interchange on $\mathrm{A}$.

[3.2] $\mathrm{V}(\mathrm{K}):=\operatorname{SUM}\left(\mathrm{B}(\mathrm{I})^{*} \mathrm{~V}(\mathrm{I})\right) / \mathrm{X}$

Then the $\mathrm{V}(\mathrm{I})$ are still linearly independent over $K(X)$ and have poles no worse than $D$ at $P$.

[3.3] Sort the $V(I)$ as in [1].

[3.4] Recompute $\mathbf{A}(\mathbf{K}, \mathbf{J})$ as in [2].

[4] Return V. 


\section{Proof of Algorithm [1] - [3]}

Let us first introduce some notation. Assume $K$ is an algebraic field, and let $L$ be its algebraic closure. Let $F(x, y)$ be the defining equation of our algebraic curve* whose coefficients lie in $K$, and let it have degree $n \geq 1$ in $y$. We assume that $F$ is irreducible over $L$. Furthermore, let it have $n$ distinct unramified places $Q_{i}$ lying over infinity. Let $R$ be the field $\{L(x, y) \mid F(x, y)=0\}$. Suppose we are given $s \geq 1$ distinct elements of $L, a_{h}$ $(1 \leq h \leq s)$, and we denote by $A_{h i}\left(1 \leq i \leq r_{h}\right)$ the places ${ }^{+}$of $R$ lying over the (finite) point $x=a_{h}$ and we denote their ramification indices by $e_{h i}$ respectively, so that $\sum_{i=1} e_{h i}=n$. Finally we assume that we are given positive integers $v_{h i}$ for each $A_{h i}$, and we let $M$ be the set of all $g$ whose order at each $A_{h i}$ is $\geq-v_{h i}$ and whose order at each other place of $R$ is $\geq 0$ (including infinite places). Hence we are regarding the $A_{h i}$ and the $v_{h i}$ as a divisor, and $M$ as the set of functions with divisor greater than this divisor of poles.

If $a$ is any member of $L$, and $A_{i}(1 \leq i \leq r)$ are the places of $R$ lying over the point $x=a$, (with ramification indices $e_{i}$ respectively) we associate with the $A_{i}(1 \leq i \leq r)$ integers $v_{i}$ $(1 \leq i \leq r)$ as follows: If $a$ is one of the $a_{h}$ mentioned in the previous paragraph, then $v_{i}=v_{h i}$, otherwise we let $v_{i}$ be 0 . We will write $\sum_{i=1}^{r} v_{i}=V$. Now let $M^{\prime}$ be the set of all $g$ in $R$ whose order at $A_{i}$ is $\geq-v_{i}$ for all elements $a$ of $L$. Thus $M^{\prime}$ is an $L[x]$ module of dimension $n$. The first step in the construction of a basis for $M$ over $L$ will be the construction of an integral basis of $M^{\prime}$ over $L[\mathrm{x}]$, where elements $w_{1}, \ldots, w_{n}$ of $M^{\prime}$ are said to form an integral basis of $M^{\prime}$ over $L[x]$ iff they are linearly independent over $L[x]$ and every element of $M^{\prime}$ can be expressed an a linear combination of them with coefficients from $L[x]$.

Let $w_{1}, \ldots, w_{n}$ lie in $M^{\prime}$. We can write the expansion of $w_{j}$ at $A_{i}$ as $w_{j}=X^{-v} \Sigma w_{j i k} x_{k}$

* This does not preclude the use of multivariate representations for computational purposes; we merely choose to state and prove these results about Coates' Algorithm in a primitive representation. My code uses multivariate representations throughout its work on Coates' Algorithm.

+ Which Coates refers to as valuations in his paper (1970). As far as we are concerned, the two words are interchangeable. 
where $X^{e}=(x-a)$ and $X$ is therefore a local parameter at $A$ with ramification index $e$. Let $D(a)$ be the determinant whose $j$-th row consists of the elements $w_{j i k}$ with $1 \leq i \leq r$ and $0 \leq k<e_{r}$ arranged in lexicographic order of indices.

Lemma 1 (this is equivalent to Lemma 6 of Coates(1970)) $w_{1}, \ldots, w_{n}$ form an integral basis of $M^{\prime}$ iff $D(a)$ is non-zero for all $a$ in $L$.

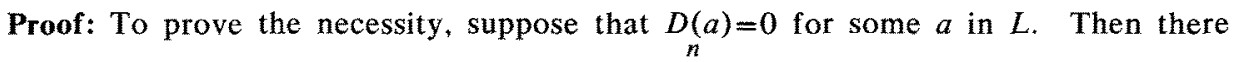
exist elements $x_{1}, \ldots, x_{n}$ of $L$, not all zero, such that $\sum_{j=0}^{n} w_{j i k} x_{j}=0$ for $1 \leq i \leq r$ and $1 \leq k<e_{i}$, since the rows of the matrix must be linearly dependent over $L$, but then $w=(x-a)^{-1} \sum_{j=1}^{j=n} x_{j} w_{j}$ belongs to $M^{\prime}$, and hence such that the original $w_{j}$ were not an integral basis.

Now let us assume that $D(a)$ is always non-zero, and then prove the sufficiency of this condition. Let $d\left(w_{1}, \ldots, w_{n}\right)$ be the determinant whose $i j$-th element is $w_{i}^{(j)}$, where this stands for one of the $n$ field conjugates of $w_{i}$ belonging to an extension of $L[x]$ in which $F(x, y)$ splits into linear factors and the conjugates are ordered suitably*. Then $d\left(w_{1}, \ldots, w_{n}\right)$ is an element of $L(X)$ and is not identically zero, for if we choose $a$ so as to have $n$ unramified places above it, we have that $d\left(w_{1}, \ldots, w_{n}\right)=D(a)(x-a)^{V}+$ higher powers of $(x-a)$ (since the expansion of $w_{i}$ at $A_{j}$ is the formal power series in $(x-a)$ representing $\left.w_{i}^{(j)}\right)$ and $D(a)$ is non-zero. Since this is so, the $w_{i}$ form a basis for $M^{\prime}$, and any $w$ in $M^{\prime}$ can be written uniquely as $w q(x)=\sum_{j=1} q_{j}(x) w_{j}$, where $q(x)$ and the $q_{j}(x)$ are polynomials in $x$ with no common factor. If we can show that $q(x)$ is a constant, then we will have expressed $w$ integrally in terms of the $w_{j}$ and the lemma will be proved. So we suppose, if possible, that $q(x)$ has a factor $(x-b)$. Then write $q(x)=(x-b) q^{\prime}(x)$ and consider

\footnotetext{
* In fact, $d$ is only determined to within a factor of \pm 1 , since the determinant can be permuted, but this will not worry us. Coates (1970, Lemma 6 ) defines $d$ to be the square of the determinant in order to avoid this ambiguity, but we will not do so. I am grateful to Dr. Norman for pointing out this simplification.
} 


$$
z=\frac{\sum_{i=1}^{i=r} q_{i}(b) w_{i}}{x-b}=q^{\prime}(x)-\frac{\sum_{i=0}^{i=r} q_{i}(x)-q_{i}(b)}{x-b}
$$

Now each term of the last expression belongs to $M^{\prime}$, so $z$ belongs to $M^{\prime}$. But the $q_{i}(b)$ are constants, not all zero (for else $(x-b)$ would be a common factor of all the $q$ 's, contrary to hypothesis) and $D(b)$ is non-zero, so there is a combination $(i, k)$ with $k<e_{i}$ such that $\sum_{j=0} q_{j}(b) w_{j i k}$ is non-zero. Therefore $z$ cannot lie in $M^{\prime}$ - the required contradiction.

This Lemma suggests the idea of a reduction step, which consists of replacing one of the $w_{i}$ in the basis by the $w$ computed in the first portion of the Lemma (equation 1), thus giving a new basis $w_{1}^{\prime}, \ldots, w_{n}^{\prime}$ which is "closer" to being an integral basis than the initial basis. The algorithm INTEGRAL_BASIS_REDUCTION described above performs as many of these reduction steps as are needed over the point $P$, having chosen, for computational convenience, a model of the curve over which $P$ is unramified.

Lemma 2 The process terminates after a finite number of reduction steps.

Proof: We first observe that, after a reduction step at $a, d\left(w_{1}, \ldots, w_{n}\right)=$ $d\left(w_{1}^{\prime}, \ldots, w_{n}^{\prime}\right)(x-a)$ to within a constant factor (i.e. an element of $L$ ) since we have replaced one of the $w_{i}$ by an element with an extra factor of $(x-a)$ in the denominator. Now let $A(x)=\prod_{h=1}\left(x-a_{h}\right)^{v_{h i}}$ Then the elements of $A(x) M^{\prime}$ have no finite poles at all, and in fact $d\left(w_{1}, \ldots, w_{n}\right) A(x)$ lies in $L[x]$, since each element in the sum which is the determinant contains one item from each conjugate, and therefore can only contain one contribution of each $v_{h i}$ for each $h$. Then, since $d$ is decreasing with each reduction step and is bounded below, the process must terminate.

Proof of Algorithm [4] - [5]

We have now constructed an integral basis for the space $M^{\prime}$, say $\left(w_{1}, \ldots, w_{n}\right)$. Let the expansion of $w_{j}$ at $Q_{i}$ (which are the places over infinity) be given by $w_{j}=X_{k=0}^{l} \sum_{k=0}^{\infty} w_{i j k} X^{k}$ 
where $X=1 / x$ is a local parameter at $Q_{i}$ since it is unramified and $l_{j}$ is an integer such that $w_{i j 0}$ is non-zero for some place $Q_{i}$ (although not necessarily for all such). The basis is defined to be a normal integral basis if the determinant $D$, whose elements are defined to be $w_{i j 0}$, is non-zero. We will assume that the $w_{i}$ have been ordered such that $l_{i} \geq l_{i+1}(1 \leq i<n)$ and that the $l_{i}$ are non-negative for $i \leq l$ (it may be that $l=0$, of course).

This suggests the idea of a reduction step at infinity, in which, if $D=0$ so that there are $x_{1}, \ldots, x_{n}$ with $\sum_{j=1} x_{j} w_{i j 0}=0(1 \leq i \leq n)$, and we let $x_{h}$ be the last non-zero $x_{i}$, we replace

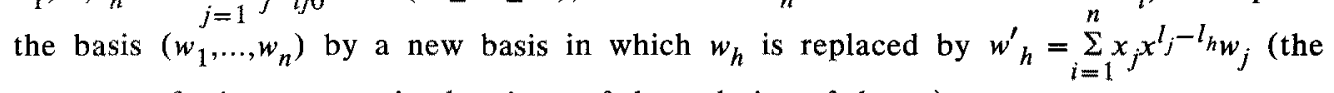
exponent of $x$ is non-negative by virtue of the ordering of the $w_{i}$ ).

Lemma 3 If the $w_{i}$ form an integral basis of $M^{\prime}$, applying a reduction step at infinity leaves them an integral basis.

Proof: It is clear that the new set of $w_{i}$ lie in $M^{\prime}$, so the only question is whether or not they form an integral basis. But a reduction step at infinity does not alter $d\left(w_{1}, \ldots, w_{n}\right)$ (except by a constant multiple), since $w_{h}$ is replaced by a linear combination of rows, in which $w_{h}$ appears with constant coefficient.

Lemma 4 We can only perform a finite number of reduction steps at infinity.

Proof: After a reduction step at infinity, $l_{h}$ has increased by at least 1 , and the rest of the $l_{i}$ are unchanged (since the corresponding $w_{i}$ are). Therefore $\sum_{i=1} \sum_{i=n}$ increases by at least 1 on each reduction step over infinity. Conversely $\sum_{i=1} l_{i}$ is at most equal to the order of $d\left(w_{1}, \ldots, w_{n}\right)$ at infinity (since $w_{j}^{(i)}$ has order at least $l_{j}$ at any of the $Q_{k}$ ) and, as remarked in the previous Lemma, $d\left(w_{1}, \ldots, w_{n}\right)$ is unchanged after a reduction step, so $\sum_{i=1} l_{i}$ is bounded above.

Lemma 5 (this is equivalent to Lemma 9 of Coates (1970)) If $w_{j}(1 \leq j \leq n)$ is a normal integral basis of $M^{\prime}$, then $\left\{x^{h} w_{j} \quad 1 \leq j \leq l \quad 0 \leq h \leq l_{j}\right\}$ is an $L$-basis of $M$.

Proof: Clearly the elements of this set are linearly independent over $L$, and lie in $M$, so all that remains to be proved is that, for any $g$ in $M, g$ can be expressed as an $L$-linear 
combination of these elements. Since $g$ is in $M^{\prime}$, we can write that $g=\sum_{j=1}^{j=n} q_{j}(x) w_{j}$ where $q_{j}(x)$ lies in $L[x]$, since the $w_{j}(1 \leq j \leq n)$ form an integral basis of $M^{\prime}$. Let $m_{j}$ be the degree of $q_{j}$ as a polynomial in $x$, and $m$ be $\min \left(l_{j}-m_{j}\right)$ over all $j$ with $q_{j}(x)$ not identically zero. Thep we can write $q_{j}(x)$ as $c_{j}(1 / x)^{m-l_{j}}+$ higher powers of $(1 / x)$. Hence, at $Q_{i}, g$ is $(1 / x)^{m} \sum_{j=1}^{m} c_{j} w_{i j 0}+$ higher terms in $1 / x$. Since $D$ is non-zero, at least one of these $\Sigma c_{j} w_{i j 0}$ is non-zero. Then the order of $g$ at this $Q_{i}$ is $m$. Since $g$ belongs to $M$, we can deduce that $m \geq 0$, and hence that $m_{j} \leq l_{j}$ which proves that $g$ lies in the space generated by the set.

\section{Extensions}

The version of Coates' Algorithm described above only works for divisors lying wholly over finite points. This is clearly not a fundamental restriction, and many ways could be devised to circumvent it. The most obvious way is to transform the curve, and the space in which it lies, so that the line (or hyper-plane, in general) at infinity no longer passes through any of the places of the divisor. The trouble with this is two-fold: such a transformation is quite difficult when we have more than one algebraic quantity; and it introduces a wholly fictitious set of algebraic entities. Even in view of the remark in the previous chapter under "Algebraic Necessities", which says that these can (and should) be kept distinct from the previous ones, we will still have some work to do to establish irreducibility and to compute Puiseux expansions. 
The technique now adopted in the program avoids both these difficulties. Let $N$ be the greatest (i.e. most negative) order at infinity. Then add $N$ to all the orders at infinity (making allowances for any ramification that may be present) and subtract $N$ from all those lying over $0^{*}$. All our poles now lie over finite places, and we can apply Coates' Algorithm in the form stated above. We then multiply all the answers by $X^{N}$, and have a basis as required, except that it will be for functions with poles of order $N$ over all infinite places. Reducing these orders to the ones we want is merely a matter of applying some linear constraints, so that a basis for the functions with at worst the desired poles is easily achieved.

We have only considered divisors of poles so far. However, a major use of Coates' Algorithm is to determine whether or not a divisor is the divisor of a function, and for this we must find a basis (which will necessarily be 1-dimensional if it exists) for the functions with the zeros and poles of the divisor. The obvious way, and the one I used to adopt, is to take the basis of functions with at worst the poles from the divisor (as found by the algorithm COATES) and then to regard the zeros as a series of linear constraints ${ }^{*}$, and to solve the resulting set of linear equations. One might think that this was not guaranteed to work, because not all the functions in the basis produced by Coates' Algorithm have all the poles, so why should that linear combination of them which has the zeros have all the poles? In fact, if the divisor were of degree 0 (and otherwise it cannot be the divisor of a function), then any function produced which has all the zeros must also have all the poles, because it must have that number of poles and it can have no others, since no element in the basis has any others.

This solution works very well in most cases, but there are difficulties of a practical nature which can arise. Consider the problem in $K(X)$ of finding the function with a zero

\footnotetext{
* It is possible that there may not have been any places lying over $X=0$ in the divisor, in which case we have to insert (with multiplicity 0 ) all the places on the curve lying over $X=0$. It might be more efficient to choose a value other than $X=0$, to avoid having to do reduction over an extra value of $X$, but $I$ have not done this.

\# These constraints are of the same type as those which were introduced in the previous paragraph to deal with the movement of poles from infinity, and so we only need to solve one set of linear equations, rather than two.
} 
of order 1000 at $X-1$ and a pole of order 1000 at $X$ (which is clearly $\left(\frac{X-1}{X}\right)^{1000}$ ). Our basis for the functions with a pole of order $\leq 1000$ at $X$, and no poles elsewhere, could be $1,1 / X, 1 / X^{2}, \ldots, 1 / X^{1000}$. We then have a 1000 by 1000 set of linear equations to solve in order to find the correct function. Even though the derivation of these equations suggests that they must have a great deal of structure, straightforward calculation makes them dense (because the solution is the row vector whose $i$-th element is the binomial coefficient ${ }_{1000} C_{i}$, and which has no non-zero entries) and therefore impractical to solve. This particular difficulty could be solved by adapting Coates' Algorithm to deal with zeros as well as poles (a trivial task) and starting off with the function $(X-1)^{1000}$, but this solution does not work for general algebraic problems. Consider the curve defined by $F(X, Y)=Y^{2}-\left(X^{2}+1\right)$, and try to find the function with a pole of order 1000 at one place over $X$, and a zero of order 1000 at the other (the answer is $\left(\frac{X}{Y-1}\right)^{1000}$ as the reader may verify). Here our initial guess would have to be $X^{1000}$ and we would then have to perform 2000 reduction steps over $X$ and the intermediate basis would contain dense polynomials of order 2000 in $X$. These examples with large numbers are given to illustrate that this algorithm and the work based on it suffer very badly from exponential growth.

The following alternative technique ${ }^{\#}$ (which has now been implemented in the latest version of the integration system) removes all these difficulties. Suppose we wish to find a function with poles at $P_{1}, \ldots, P_{n}$ and zeros at $Q_{1}, \ldots, Q_{n}$. Let $f$ be a function with poles at $P_{1}$ and $P_{2}$ and a zero at $Q_{1}$. Such a function* can readily be found by Coates' algorithm. Then let $P^{\prime}$ be the other zero of $f$, and $f^{\prime}$ be the function (found recursively) with poles at $P^{\prime}, P_{3}, \ldots, P_{n}$ and zeros at $Q_{2}, \ldots, Q_{n}$. Then the answer to the original problem is $f^{*} f^{\prime}$.

We give below a definition of this algorithm, and note that, for a constant curve and constant places over which the divisor lies, its running time is (approximately) proportional to (number of places) $* \log _{2}$ (largest multiplicity).

\# I am very grateful to Professor Sir Peter Swinnerton-Dyer for suggesting this approach.

* If it exists - see Steps [2.2.1] and [2.4.1] below, and the discussion after the algorithm. 


\section{DIVISOR TO FUNCTION}

Input: $\mathrm{F}(\mathrm{X}, \mathrm{Y})$ : The equation of an algebraic curve

(possibly in a multivariate representation).

D: a divisor of degree 0 on that algebraic curve.

Output: FUN: a function on that curve

which has that divisor as its divisor of zeros and poles, or FAILED if there is no such function.

[1.1] POLES: = All the poles in D, with appropriate multiplicities.

ZEROS:= All the zeros in D, with appropriate multiplicities.

[1.2] FUN: $=1$.

In FUN we accumulate the answer as we reduce the divisor $D$.

[2] While (number of ZEROS) $>1$ do:

[2.1] P1:= Any member of POLES.

P2: = Any member of (POLES - P1).

Note that P1 and P2 could be the same, if a pole occurs in POLES with multiplicity $>1$. In theory it does not matter which elements of POLES we choose, but in practice the running time of the algorithm is decreased if we choose $\mathrm{P} 1$ and $\mathrm{P} 2$ such that the divisor $\mathrm{P} 1+\mathrm{P} 2$ divides POLES as often as possible, and this can be accomplished by letting $P 1$ be the element of POLES of greatest multiplicity, and P2 that element of POLES' of greatest multiplicity, where POLES' is POLES with the multiplicity of P1 halved (rounding down).

[2.2] $\mathrm{V}:=\operatorname{COATES}(\mathrm{F}(\mathrm{X}, \mathrm{Y}),-\mathrm{P} 1-\mathrm{P} 2)$.

\section{[2.2.1] If $\mathrm{V}=$ FAILED}

Then go to [4]. 
[2.3] Z:=an element of ZEROS

Here again it does not matter which element we choose, but the running time of the program is improved by choosing an element of greatest multiplicity.

[2.4] $\mathrm{V}:=$ That linear combination of elements of $\mathrm{V}$ with a zero at $\mathrm{Z}$.

[2.4.1] If $\mathrm{V}=$ FAILED

Then go to [4].

$[2.5] \mathrm{Z}^{\prime}:=$ The other zero of $\mathrm{V}$.

[2.6] While (P1+P2) | POLES and $\mathrm{Z} \mid$ ZEROS do:

[2.6.1] POLES:=POLES-P1-P2.

[2.6.2] ZEROS: $=$ ZEROS $-\mathrm{Z}$.

[2.6.3] If $\mathrm{Z}^{\prime} \mid \mathrm{ZEROS}$

Then ZEROS: $=$ ZEROS $-Z^{\prime}$

Else POLES: $=$ POLES $+Z^{\prime}$.

$[2.6 .4]$ FUN: $=$ FUN*V.

[3] If ZEROS is null

Then return FUN.

[4] (Final step)

We come here when we have some POLES left that we cannot explain away in pairs by the operation of [2.6]. This could occur either because there is only one element of POLES left (the usual case), or because one of [2.2] and [2.4] returned a FAILED (the exceptional case). The exceptional case is discussed at greater length below.

[4.1] $\mathrm{V}:=\operatorname{COATES}(\mathrm{F}(\mathrm{X}, \mathrm{Y}),-\mathrm{POLES})$. 


\section{[4.2] If ZEROS = zeros of $\mathrm{V}$}

\section{Then return FUN*V}

Else return FAILED.

As mentioned at [4], there is no need for the intermediate elements of FUN to exist at all, even when the final problem* has a solution. This problem occurs, basically, because there might be no functions with only 2 poles (though this can't happen on curves of genus 1). We can continue by using the ordinary COATES procedure, as outlined in steps [4.1] and [4.2], but perhaps there is scope for a more enlightened process of adding poles, one at a time, until the space of functions is 2-dimensional, then taking a combination of these functions to kill a zero from the divisor, and repeating, but $I$ have no idea how well this would behave in practice. It is quite clear that this chapter on Coates' algorithm has barely scratched the surface of what should become a fruitful area for further investigation by computer algebra workers.

\section{Coates' Algorithm \& Differentials}

We can apply Coates' Algorithm to differentials, as well as to functions. We can write a differential as $F(X, Y) \mathrm{d} X$ for some function $F$, and we need then to consider what our local parameters are at each place on the curve. There are three possibilities for the point over which our place lies:

1) The place could lie over infinity.

* The following example (for which I am grateful to Professor M.F. Singer) shows this. Let $C$ be a curve of genus $g \geq 3$ which is not hyper-elliptic (i.e. cannot be represented as a 2-sheeted cover of the projective line, which essentially means that it is not of the form $y^{2}=p(x)$ ). Let $f$ be a rational (non-constant) function on $C$ with the minimal number $k$ of zeros and poles. Then, since $C$ is not hyper-elliptic, it follows that $k>2$ (see any standard book on algebraic curves). Hence there is no rational function on $C$ with only two poles, and an application of DIVISOR_TO_FUNCTION to $(f)$ would fail. 
In that case our local parameter is $t=(1 / X)^{1 / k}$ for some integer $\mathrm{k}$, so $\mathrm{d} t / \mathrm{d} X=t^{-(k+1)}$, so our differential will have order $k+1$ less at infinity than the function $F$ does.

2) The place could lie over a point a which is a root of $Y$.

In this case our local variable is $t=(X-a)^{1 / k}$ for some integer $k$. This gives us $\mathrm{d} X / \mathrm{d} t=t^{k-1}$, so the differential has order $k-1$ more than the function.

3) The point can have neither property.

In this case $(X-a)$ is a local parameter, and the differential has the same order as the function.

Thus, in order to find a basis for the $K$-space of differentials with poles no worse than a specified divisor, we only need to adjust the multiplicities in the divisor at the (finite number of) places in categories 1 and 2 above, and then apply Coates' Algorithm to find a basis for the functions $F(X, Y)$.

In particular, we can find the genus of an algebraic curve, since it is the dimension of the space of differentials of the first kind, i.e. those with no poles. The divisor obtained from the zero divisor by applying the manipulations described in 1) and 2) above is termed the canonical divisor of the curve.

\section{Implementation}

Since Coates' Algorithm is going to be a fundamental building block in our integration algorithms, it is going to be worth our while to consider the implementation of this rather carefully. One particular technique of importance is the minimisation of the number of Puiseux expansions calculated in the course of Coates' Algorithm. In particular we need not recompute the Puiseux expansion of $A+B$ from scratch if we know the Puiseux expansions of $A$ and $B$, but we can use the identity which says that the taking of Puiseux expansions commutes with addition.

During the operation of the algorithm INTEGRAL_BASIS_REDUCTION, it may 
happen that one entire row of the matrix $A(I, J)$ is zero. If the I-th row is zero, then we can immediately deduce that $\mathrm{V}(\mathrm{I})$ should be replaced by $\mathrm{V}(\mathrm{I}) /(\mathrm{X}-\mathrm{P})$, and we can do this and recompute the $\mathrm{I}$-th row of the matrix. Furthermore we need not re-expand this new function about the different places, but we can take the original Puiseux expansions and simply shift the power series by one term in order to produce the Puiseux expansions of the new V(I). This is especially suitable given our method for computing Puiseux expansions described in Chapter 2, since new terms can be computed at any stage while still making use of all previously computed partial results.

We may, having tried Coates' Algorithm on the divisor $k D$, wish to try it on $(k+1) D$. A naive application of the Algorithm COATES above (not using DIVISOR_TO_FUNCTION) would start each time from the same initial basis in step [1] of Algorithm COATES, thus throwing away in the computation of $(k+1) D$, all the information gained from the computation of $k D$. In fact we can start step [2] with the normal integral basis from step [4] of the $k D$ attempt, since this basis is a set of $n$ linearly independent members of $M^{\prime}$. The advantage of this is that these members are much closer to having poles specified in $(k+1) D$ than the elements that would be computed in step [1], in particular they have the poles of $k D$. If $D$ has total degree $d$, then the number of poles (counted with degree) that have to be introduced into the basis in the computation of Coates' Algorithm for $D, 2 D, \ldots, k D$ is $k d$ rather than the $k(k+1) d / 2$ that the obvious algorithm would require. There is an illustration of this in Chapter 5 ,under the heading Cayley's method, where it is shown that (using COATES rather than DIVISOR_TO_FUNCTION) a saving of 5.3 seconds can be made on a time of 38.2 seconds by this technique.

If we are using DIVISOR_TO_FUNCTION, then we can restart at the end of step [3] and add the poles and zeros of $D$, which is the difference between $k D$ (whose function we had almost finished computing) and $(k+1) D$ (whose function we wish to compute). 


\section{Conclusions}

There are two main application areas for the algebraic geometric treatment of expressions outlined in this chapter and the previous one. The first is the integration of algebraic functions, which is the main subject of this work. The second is concerned with locating integer (or rational number) solutions to sets of algebraic equations. Much work has been done on this (see the survey Swinnerton-Dyer,1976), but so far it has been approached by ad hoc arithmetical techniques, with all the algebraic geometry translated into FORTRAN (say) by hand.

We have raised the problem of finding all the functions with a specified set of poles, which is trivial for rational functions, and described Coates' Algorithm, which can provide a solution to it. This then leads to answers to the questions "does this divisor correspond to a function", "what is the genus of this curve" and "what are the differentials of the first kind". Later we will see that these are precisely the questions that are asked in the process of integrating algebraic functions. 